University of Nebraska - Lincoln

DigitalCommons@University of Nebraska - Lincoln

$11-1953$

\title{
STUDIES IN QUANTITATIVE PALEONTOLOGY. III. AN APPLICATION OF SEQUENTIAL ANALYSIS TO THE COMPARISON OF GROWTH STAGES AND GROWTH SERIES
}

\author{
Benjamin H. Burma \\ University of Nebraska-Lincoln
}

Follow this and additional works at: https://digitalcommons.unl.edu/geosciencefacpub

Part of the Earth Sciences Commons

Burma, Benjamin H., "STUDIES IN QUANTITATIVE PALEONTOLOGY. III. AN APPLICATION OF SEQUENTIAL ANALYSIS TO THE COMPARISON OF GROWTH STAGES AND GROWTH SERIES" (1953). Papers in the Earth and Atmospheric Sciences. 328.

https://digitalcommons.unl.edu/geosciencefacpub/328

This Article is brought to you for free and open access by the Earth and Atmospheric Sciences, Department of at DigitalCommons@University of Nebraska - Lincoln. It has been accepted for inclusion in Papers in the Earth and Atmospheric Sciences by an authorized administrator of DigitalCommons@University of Nebraska - Lincoln. 


\title{
STUDIES IN QUANTITATIVE PALEONTOLOGY. III. AN APPLICATION OF SEQUENTIAL ANALYSIS TO THE COMPARISON OF GROWTH STAGES AND GROWTH SERIES ${ }^{1}$
}

\author{
BENJAMIN H. BURMA \\ University of Nebraska
}

\begin{abstract}
Detailed directions for carrying out a comparison of characters of fossils by a sequential analysis are given. The method is adapted to comparison at a single growth stage and to the comparison of growth series. The method has the advantage of, in general, requiring fewer specimens than the classical methods of comparison.
\end{abstract}

An essential problem in paleontology is the comparison of two collections, or samples, and the decision as to whether these samples represent one or two species. In previous papers ${ }^{2}$ some aspects of these problems have been discussed and suggestions brought forward as to ways of making these comparisons quantitatively and of making these decisions in as objective a manner as possible. These methods have involved a quantitative or, if you please, a statistical approach. One difficulty involved in gaining acceptance for these methods is that most of them necessitate the use of reasonably large samples before decisions can be rendered certain to a high degree of probability, even in cases where the two samples are quite different. This, of course, is in marked contrast to the usual, intuitive, approach to the same problem. If a single specimen differs enough from a known species, we have little hesitation in announcing that the specimen does not belong to that species. Similarly, if a single specimen is similar enough to a given species, we are usually satisfied with the identity of the two. Conventional statistical methods are poorly adapted to such procedures. In fact, the sample size is a

\footnotetext{
${ }^{1}$ Manuscript received May 6, 1953.

${ }^{2}$ Part I of this series.
}

factor bulking very large in establishing the reliability of decisions under whatever circumstances, resulting in sample sizes being relatively fixed and necessitating the routine use of comparatively large samples to obtain dependable results.

The necessity of always using samples of this more or less fixed size can, for some purposes, be obviated by the use of sequential analysis. Sequential analysis was developed during the late war for quality control in manufacturing and other inspections. Its development was begun in March and April, 1943, by A. Wald and was so successful in wringing a maximum of reliable conclusions from a minimum of data that the whole subject was classified as "Restricted" within the meaning of the Espionage Act. This classification was finally removed in May, 1945. A report discussing some of the applications of this method has been published (Statistical Research Group, Columbia University, 1945), and also a book on the theoretical aspects (Wald, 1947).

All the methods of sequential analysis may be summarized as follows: given certain standards of comparison, the analysis of the sample in question begins. The first specimen is compared to the 
standard, and a decision is made as to whether the hypothesis in question is to be accepted or rejected or whether the data are insufficient. If one of the former two, the test is stopped; if the latter one, the test continues. The next specimen is studied, and then these two are together compared to the standard, and again a decision is made as to whether the hypothesis is to be accepted or rejected, or whether the data are insufficient. If one of the former two, the test terminates. If it is the latter, the test continues for specimen after specimen until a definite decision for or against the hypothesis is reached.

Thus, instead of using a sample of more or less fixed size, as in classical statistics, we are able to use a sample of variable size, the size depending on the developing characteristics of the sample as it is studied. This results in a great saving in the number of specimens which must be studied, as compared to the classical methods. In the method described below it may be expected that, on the average, a decision will be reached with a sample only about half the size of the sample needed to reach the same decision by the classical methods. Note that this saving is true on the average. Again, in the method discussed below, it may be expected that in about 5 per cent of the cases a sample as large as or larger than the classical sample size would be necessary to reach a decision, but the probability is negligible that a sample as much as twice as large as the classical sample would be necessary. Even with this being true, it is still to be expected that a saving of some 50 per cent in sample size will be effected over-all. Time savings will be even greater, since the calculations used in sequential analysis are extremely simple, and a graphical method is used to indicate whether at any stage the test is to go on or whether one decision or the other has been reached.

The methods of sequential analysis are so new that they are still in the process of active development. At the present, only one method of wide application to paleontology seems to be available. This is a method suitable for comparing a given character of one sample with this same character in another sample. The decision to be reached is to be whether the two samples are, with respect to this character, statistically the same, with the implication that the two samples may represent one species, or whether they are different, which would imply that they belong to different species. (For a discussion of these implications, see part I of this series of papers.) The directions for using this method will now be given in some detail. This method is adapted from section 5 of the report of the Statistical Research Group. The tables given are revised and extended from that section. The method given immediately below is suitable only for comparing samples which are of the same growth stage. Comparison of growth series is discussed subsequently.

\section{THE COMPARISON OF GROWTH STAGES}

Perhaps the best way to make the directions for this method clear is to follow through an actual problem. I have chosen one dealing with a comparison of three species within the fusulinid genus Triticites with a fourth, T. caccus. Three characters of this species are to be compared individually with each of the other three species. Only one of these need be followed through in detail; the rest will simply be shown graphically at the end. For this purpose, we will compare the form-ratio of the fifth volution of $T$. collus with the form-ratio of the fifth 
volution of our "standard," T. caccus. Other quantities used:

In order to make this comparison, we must know certain things concerning this form-ratio of $T$. caccus, both to set up the graphical framework for the comparison

$x$-an observation on the variable (the formratio of $T$. collus).

$n$-the number of observations.

$\left|\Sigma\left(x-m_{1}\right)\right|$-the absolute value of the sum of the differences of the observed values of $x$

TABLE 1

VALUES OF $c$ IN TERMS OF $\gamma(c=\ln [1-\gamma] / \gamma)$

$\gamma=0.000-0.049$

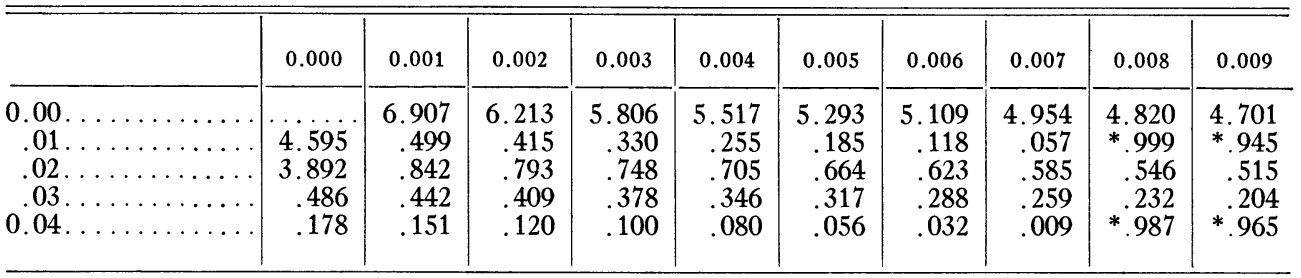

$\gamma=0.000-0.49$

\begin{tabular}{|c|c|c|c|c|c|c|c|c|c|c|}
\hline & 0.00 & 0.01 & 0.02 & 0.03 & 0.04 & 0.05 & 0.06 & 0.07 & 0.08 & 0.09 \\
\hline $\begin{array}{r}0.0 \ldots \\
.1 \ldots \\
.2 \ldots \\
.3 \ldots \\
0.4 \ldots\end{array}$ & $\begin{array}{r}2.197 \\
1.386 \\
0.846 \\
.405\end{array}$ & $\begin{array}{r}4.595 \\
.091 \\
.324 \\
.802 \\
.365\end{array}$ & $\begin{array}{r}3.892 \\
* .992 \\
.267 \\
.756 \\
.322\end{array}$ & $\begin{array}{r}3.486 \\
* .901 \\
.209 \\
.708 \\
.285\end{array}$ & $\begin{array}{r}3.178 \\
* .798 \\
.154 \\
.663 \\
.239\end{array}$ & $\begin{array}{r}2.944 \\
* .735 \\
.099 \\
.615 \\
.199\end{array}$ & $\begin{array}{r}2.751 \\
* .658 \\
.047 \\
.577 \\
.157\end{array}$ & $\begin{array}{r}2.594 \\
* .585 \\
. * .993 \\
.531 \\
.122\end{array}$ & $\begin{array}{r}2.441 \\
* .517 \\
* .944 \\
.489 \\
.077\end{array}$ & $\begin{array}{r}2.312 \\
* .449 \\
* .896 \\
.445 \\
.039\end{array}$ \\
\hline
\end{tabular}

and to make the actual comparison with $T$. collus. These are as follows:

$m$-the mean form-ratio of the fifth volution of T. caccus. $m=3.21$.

$d$-the deviation, the amount that the mean form-ratio of $T$. collus must depart from the mean of $T$. caccus before we will consider the two to be distinct. As shown in part I of this series of papers, we will consider this point reached when $d=3 \sqrt{2} \sigma_{m}$, where $\sigma_{m}=$ the standard error of the mean of $T$. caccus $(0.263)$. Here $d=1.10$.

$\gamma$-the risk we are willing to take of accepting the hypothesis that the form-ratios are statistically equal when they are, in fact, not equal, or of rejecting the hypothesis when they are, in fact, equal. The conventional probability in such cases is $0.003(3 \sigma)$, or 3 chances in 1,000 of making the wrong decision. Greater or lesser probabilities may be used in given problems. $\gamma=0.003$.

$\sigma$-the standard deviation of the mean of the form-ratio of the fifth volution of $T$. caccus. $\sigma=0.788$. and $m$, after $n$ observations have been made.

ln-the natural logarithm, logarithm to the base $e$.

cosh-the hyperbolic cosine.

The above values are all from the known data. The next step is to construct the diagram used in the graphical comparison of the two species, as follows:

Step 1.- In table 1 find the value of $c$ corresponding to the value of $\gamma$ above. In this case $\gamma=0.003$, and the corresponding value of $c$ is 5.806 .

Step 2.-Calculate the slope $s$, using the formula $s=d^{2} / 2 \sigma^{2}$. In this case $s=$ 0.974:

$$
s=\frac{1.10 \times 1.10}{2 \times 0.788 \times 0.788}=0.974 .
$$

Step 3.-Construct the straight lines $I$ and $I I$ of figure 1 . These two lines will cut the $y$-axis at $c$ and $-c$. The equation 
of line $I$ is $y=s x+c$, and that of line $I I$ is $y=s x-c$. In this case for line $I$, $y=0.974 x+5.806$; and for line $I I$, $y=0.974 x-5.806$. In both cases the points $c$ and $-c$ are marked off on the $y$-axis at +5.81 and -5.81 , respectively. Another value of $x$ is then substituted in the equations to get another point on each line. It is best to choose a value of $x$ as large as the graph permits, in this case, $x=15(=n)$. Thus, for line $I, y=$ ues of $x$ and $y$ are plotted on the graph. So long as these points remain between lines $I$ and $I I$, the testing is continued. If a point falls outside the area between these two lines, the testing is stopped and a decision made. If the point falls above line $I$, it signifies that the two samples are not the same, in so far as this character is concerned; if it falls below line $I I$, it signifies that the two are the same. The probability that the correct conclu-

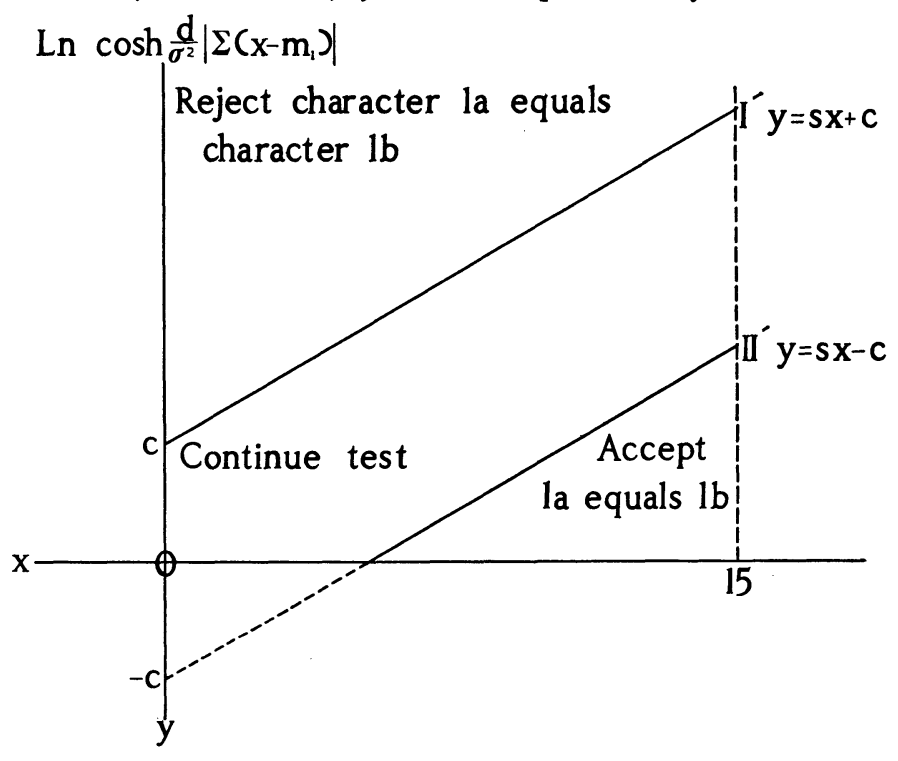

FIG. 1.-Graphical framework for the conducting of a sequential test

$0.974(15)+5.806=14.61+5.81=$ 20.42; and for line $I I, y=8.80$. Line $I$ is then drawn through the points $x=0$, $y=5.81$, and $x=15, y=20.42$; and line $I I$, through $x=0, y=-5.81$, and $x=15, y=880$.

Having constructed our graphical framework, we are ready to proceed to the actual comparison. Note that this frame can be used for comparing the formratio of the fifth volution of any other fusulinid with $\mathrm{T}$. caccus and not just the one species, T. collus. The comparison is made by calculating for each successive value of $x$ the value $y=\ln$ cosh $\left(d / \sigma^{2}\right)\left|\Sigma\left(x-m_{1}\right)\right|$. These successive val- sion has been reached in the latter two cases will be at least $1-\gamma$ (in this case $1-0.003$, or 0.997 ; 997 in 1,000$)$. Although the formula used in calculating the successive values of $y$ has an extremely formidable look, the calculation is actually made very simple by the use of a table and by breaking the calculation down into steps.

To return to our specific problem, the calculation may be carried forward conveniently in tabular form, as shown in table 2 . The headings of the columns denote the successive steps to be taken. The first specimen to be studied is taken $(n=1)$, and its form-ratio is measured 
$(x=3.36)$. These values are placed in the first two columns. For the third column, $m_{1}$ (3.21 in this case) is subtracted from $x$, keeping proper account of algebraic signs. Then $3.36-3.21=0.15$ is entered in this column. The fourth column, the successive sum of this subtraction, is next. Since this is the first figure, 0.15 is entered here also. The value of the fourth column is multiplied by $d / \sigma^{2}$, in this case by 1.77 , and the answer, 0.266 , is entered in the fifth column. The sixth column is the natural logarithm of the hyperbolic cosine (ln cosh) of the fifth column. This value is read directly from table 3 (p. 542). This table is arranged like a table of logarithms; thus $\ln$ cosh 0.26 is at the intersection of row 0.2 and column $0.06(=0.033)$. Using this table, 0.035 is entered in the sixth column. This number is then plotted on the graph (fig. 2) with $x=n=1$ and $y=0.035$ for coordinants. This point falls between the lines $I$ and $I I$, which means that we must continue testing. Therefore, we take our second specimen $(n=2)$ of $T$. collus, measure its form-ratio $(x=3.31)$, and subtract $m_{1}$ from this $\left(x-m_{1}=0.10\right)$. For the fourth column, 0.10 is added to the figure next above in the $\left(x-m_{1}\right)$ column $(0.10+0.15=0.25)$. This is multiplied by $d / \sigma^{2} \quad(1.77 \times 0.25=$ $0.443)$, and the $\ln$ cosh is taken of the result, using table 3 (ln $\cosh 0.443=$ 0.096). Then $x=n=2$ and $y=0.096$ are plotted on the graph and also fall between lines $I$ and $I I$; so we continue testing. A new situation arises in $n=3$. Here $x=2.04$, so that $x-m_{1}=-1.17$, a negative number. For the fourth column, -1.17 is added algebraically to $0.25(=-0.92)$. Then for the fifth column this is multiplied by 1.77 $(=-1.63)$. However, all the ln cosh's will be positive; therefore, we may drop the signs again at this point and record 1.63 for $n=3$ in the fifth column. Cor- rect account of signs must be kept in the second to fourth columns, inclusive. In the fifth column, all numbers are to be entered as positive. The $\ln \cosh$ is taken of 1.63 (=0.975). When $n=3, y=$ 0.975 is plotted, it is still between lines $I$ and $I I$, and so the testing goes on. Similar results are obtained for $n=4,5,6$, and 7. However, for $n=8$ we find that $\ln$ $\cosh 1.46=0.819$ falls below line $I I$. At this point we stop testing. We then know with a probability of 0.997 , that the form-ratios for the fifth volution of

TABLE 2

FRAMEWORK FOR CALCULATIONS

\begin{tabular}{r|r|r|r|r|r}
\hline \hline$n$ & $x$ & $x-m_{1}$ & $\left|\Sigma\left(x-m_{1}\right)\right|$ & \multicolumn{1}{c|}{$\begin{array}{c}d \sigma^{2} \\
\left|\Sigma\left(x-m_{1}\right)\right|\end{array}$} & $\begin{array}{c}\text { In cosh } \\
d / \sigma^{2} \\
\left|\Sigma\left(x-m_{1}\right)\right|\end{array}$ \\
\hline $1 \ldots$ & 3.36 & 0.15 & 0.15 & 0.266 & 0.035 \\
$2 \ldots$ & 3.31 & 0.10 & 0.25 & 0.443 & 0.096 \\
$3 \ldots$ & 2.04 & -1.17 & -0.92 & 1.03 & 0.975 \\
$4 \ldots$ & 3.53 & 0.32 & -0.60 & 1.06 & 0.480 \\
$5 \ldots$ & 3.06 & -0.15 & -0.75 & 1.33 & 0.704 \\
$6 \ldots$ & 2.72 & -0.49 & -1.24 & 2.20 & 1.519 \\
$7 \ldots$ & 3.13 & 0.08 & -1.32 & 2.34 & 1.656 \\
$8 \ldots$ & 3.70 & 0.49 & -0.83 & 1.46 & 0.819 \\
\hline
\end{tabular}

T. caccus and T. collus do not differ significantly. It would not be possible to reach a similar decision with such a small sample of $T$. collus by the classical methods.

In figure 2 may be seen the comparisons of $T$. collus, T. newelli, and T. primarius with $T$. caccus for three different characters. It will be noted that in most cases a decision is reached as to whether the species could or could not be distinguished on the basis of this character. In one case, more data would be necessary to bring about a decision. In all cases, nevertheless, there is at least one character in which each species definitely differs from $T$. caccus. (Comparisons except for proloculum made at the fifth volution.)

A point which should be mentioned is that, although a decision can be made 
that two samples are different on the basis of one specimen, several specimens are necessary to decide that the two are alike. This is due to the fact that part of line $I I$ lies below the $x$-axis, where values of $y$ (or ln cosh) would have to be negative. However, as already mentioned, ln cosh does not assume negative values. The exact minimum number for the latter decision will vary according to individual circumstances. In the characters above, it varies from five to seven specimens.

\section{THE COMPARISON OF GROWTH SERIES}

The foregoing discussion applies to the comparison of two samples of a given growth stage. Very often one may have available a fairly large number of specimens in a sample forming a growth series and yet have only one or two of these represent any one restricted growth stage. In such a circumstance it would obviously be advantageous to have a method in which the entire growth series could be used. If we try to do this, we are at once beset by difficulties.

The first, and perhaps most fundamental, of these difficulties is that the absolute variability of a given animal is much greater in the adult than in the young stages. For example, at a given growth stage in the youth of a certain animal, the length might vary from 10 to $18 \mathrm{~mm}$., with a mean of $14 \mathrm{~mm}$. or an absolute range in variability of $8 \mathrm{~mm}$.

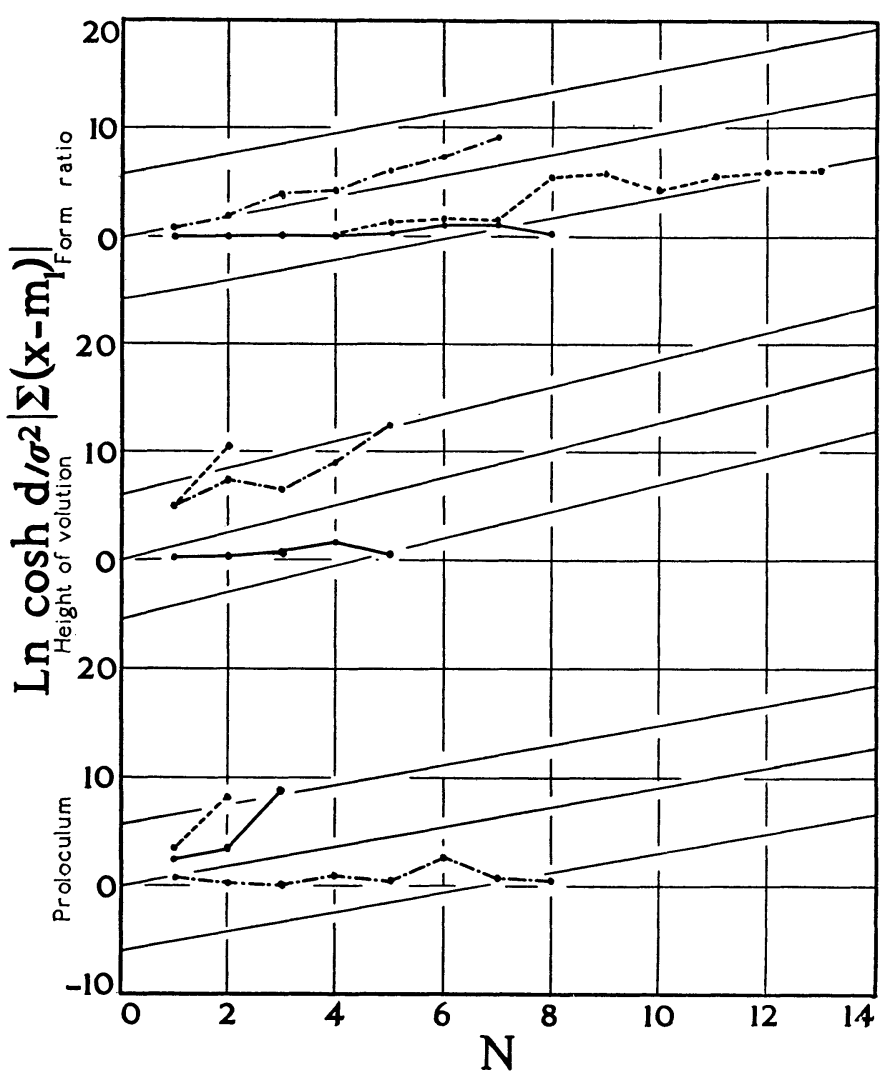

Fig. 2.-Sequential test for the comparison of Triticites caccus with $T$. collus, $T$. primarius, and $T$. newelli. Characters tested are form-ratio, height of volution (both at the fifth volution), and the external diameter of their prolocula. Solid line, T. collus test; broken line, T. primarius; dot-dash line, T. newelli. 
The same animal in an adult growth stage might vary from 40 to $72 \mathrm{~mm}$., with a mean of $56 \mathrm{~mm}$. and an absolute range in length of $32 \mathrm{~mm}$. Yet the growth stages, which have such a great difference in absolute variability, would have the same proportionate variability, in each case the range being 0.8 times the minimum. Since the size of the standard deviation depends on the absolute variability, $\sigma$ would have to appear in the sequential analysis formula as a variable rather than as a constant. This would involve recasting the formulas into a more complex form. Further, the evaluation of the function expressing the relation of $\sigma$ to the growth stages is a matter of great difficulty and is not readily soluble in absolute terms for samples of ordinary size.

This factor of the variability of $\sigma$ in absolute terms is in itself sufficient to condemn this approach to the analysis of growth series. Fortunately, another avenue is open. It will be recalled that in the last paragraph it was intimated that, while the absolute variability of a given form increases more or less constantly with an increase in age (and thus size), the proportion that the absolute variability is of, say, the minimum size remains approximately constant. Thus if we plot the growth series of a given character on an arithmetic scale, the lines bounding the maximum and minimum limits of variability will be found to diverge rapidly; but if the same data be plotted on a logarithmic scale, the lines will be very close to parallelism. I have made no attempt to obtain any exact proofs that the field of variability in the last case will be bounded by exactly parallel lines, but over a period of years I have found it to be an empirical fact that in actual practice these lines do approach parallelism within the limits of sampling errors.

If the variability of the logarithms of a growth series be constant, or nearly so, through a growth series, then it will follow that the $\sigma$ of these logarithms from the means of successive growth stages will also be a constant, and we have then sidestepped the major difficulty in the way of applying sequential, and other, tests to growth series.

Before proceeding further, however, it will probably be well to consider the propriety of using logarithms in this manner. One objection that immediately comes to mind is that, after all, animals and plants grow "by numbers," not "by logarithms." A moment's thought will reveal the shaky foundation on which this argument rests. Plants and animals do not grow "by anything." They just grow, according to natural laws, and we use numerical values to describe this growth. That we have habitually used numbers in an arithmetic series to describe this growth does not mean that the arithmetic series is essential to this growth or is even the best way to describe it. It is a matter of habit only, a very thoroughly ingrained one it is true, and one which requires a conscious effort to shed, but a habit, nevertheless. On the other hand, as was emphasized in the first paper of this series, it is an observable fact that growth is usually best defined by a power (or logarithmic) function of some sort. It can readily be argued from this that, in actual fact, logarithms are a better means of describing growth than numbers in an arithmetic series.

Granted the validity of using logarithms rather than absolute values in dealing with growth series, it remains to calculate $\sigma$ (the standard deviation) for this growth series. Since $\sigma$ depends on the variation from the mean, we have first to determine what the mean of a growth series is. It is not a simple mean obtained by adding all the values (logarithms in this case) of a certain character and di- 
viding by a number of specimens. The mean of a growth stage is the mid-point of a "field" of points on a line. The mean of a growth series is the mid-line of a field of points on a plane. The best such midline is the regression line which minimizes the square of the deviations from itself. This is readily calculated for any given set of data by usual statistical methods. This ordinary calculation of a regression line is, strictly speaking, suit- will call $P$. godoni alpha and the second $P$. godoni beta. We wish to compare the number of side plates per ambulacrum of $P . g$. beta with the number in $P$. g. alpha. The regression line is determined for the side plates of $P$. g. alpha against the standard radial as a "time" character. The graph of this regression line is shown in figure 3. Note that an arithmetic graph is used, on which the logarithms of the number of side plates and length of the

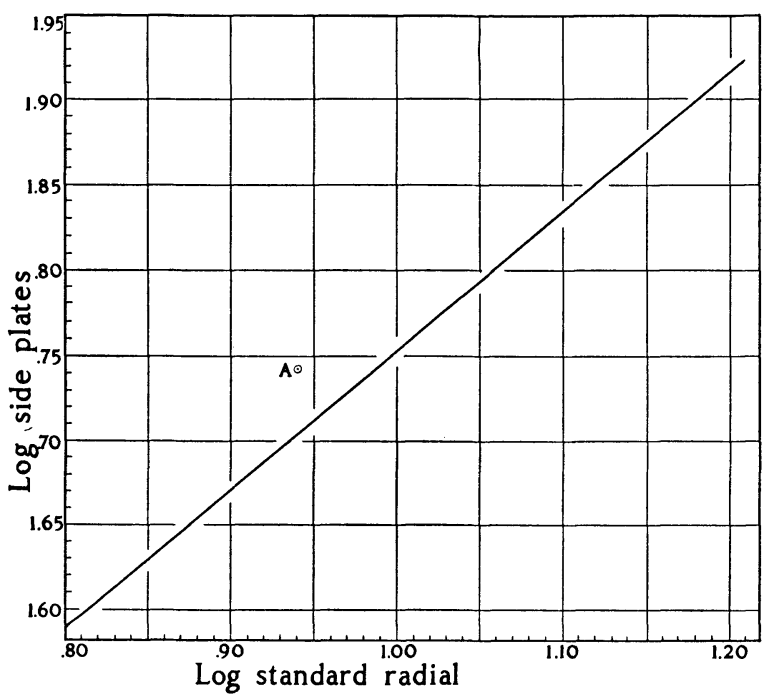

FIg. 3.-Regression line for use as the mean of a growth series

able only to a field of points having a straight-line correlation. In the majority of cases the points plotted from the logarithms will fulfil this condition. In some few cases the scatter of the points will indicate that a curved regression line would fit the data better than a straight one. In such cases a curved line may be fitted, using orthogonal polynomials. Very complete directions for this method, together with the necessary tables, will be found in Anderson and Housman (1942). In some cases this will not be necessary, and two or three straight-line segments will fit the data satisfactorily.

As an example let us use the data of two samples of Pentremites. The first we standard radial, respectively, are plotted. The actual points plotted are omitted for clarity.

The next step is to calculate the $\sigma$ for the deviation of the actual distribution of the number of side plates from this line. Since the absolute distribution of points with respect to this line is what is of interest, we may use as deviations the actual distance the plotted points are above and below the line. For example, in figure 3, point $A$ has a deviation of 0.50 inches (on the figure as originally drafted). These deviations are all measured vertically about the regression line, not at right angles to it. When the deviations of all points from the regression line 
are obtained, $\sigma$ is calculated from them in the usual manner. If the deviations are measured in inches, $\sigma$ will also be in inches. In this particular case it was 1.31 inches. This distance is plotted off vertically above any convenient point on the regression line, and the corresponding values of the log of the number of side $\gamma$-as usual, $\gamma=0.003$

$\sigma$-as calculated above, $\sigma=0.0673, \sigma^{2}=$ 0.004529 .

$s-s=d^{2} / 2 \sigma^{2}=0.4238$.

$x-m_{1}$-the difference between the number of side plates of $P$. g. beta and the mean number of side plates of $P$. g. alpha corresponding to the given standard radial of $P . g$. beta.

$(x-m)$-as in the first example.

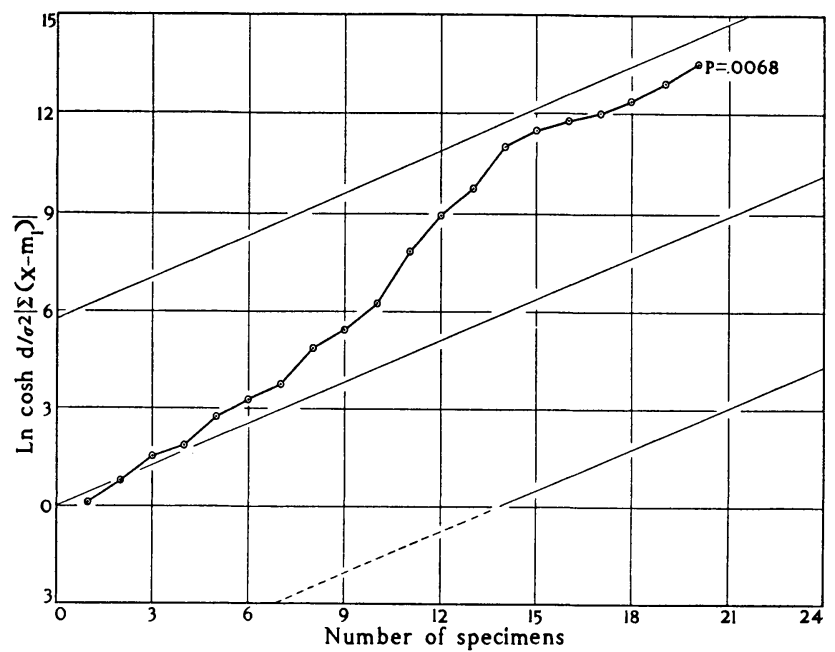
beta.

FIG.4.-Sequential test for the comparison of the number of side plates of Pentremites godoni alpha and P.g.

plates are noted for the beginning and ending of this 1.31-inch line. The smaller figure is subtracted from the larger, and the answer, 0.0673 , is the value of $\sigma$ in terms of logarithms.

We are now ready to proceed with a sequential analysis of these two samples. Our known data for the formulas are as follows:

$m_{1}$-the mean number of side plates of $P . g$. alpha corresponding to a given growth stage (standard radial) of $P . g$. beta. For example, our first specimen of $P . g$. beta has a log standard radial of 0.969 . The vertical line corresponding to this and the regression line intersect at a point corresponding to $\log$ side plates of 1.726 . This value is $m_{1}$ for the first specimen.

$d$-calculated from $\sigma_{m}$ (and eventually from $\sigma)$, as in the first example. In this case, $d=0.062$.
From this point the calculation proceeds exactly as in table 1 , except that the mean $m_{1}$ for each $n$ is separately determined, as discussed above. The progress of the test is shown in figure 4 . In the particular case we have chosen, we have only 20 specimens in our sample, which proves to be insufficient to reach a decision. The evaluation of such a case is our next problem.

\section{SIGNIFICANCE OF CURTAILED TESTS}

Sequential tests are set up on the assumption that sufficient specimens will be available to complete the test. In quality control in industry, this will ordinarily be the case. In paleontological work, however, the number of specimens available may often be insufficient to 
reach the desired conclusion. The Columbia group, in their book, describe a method for evaluating such cases, but in a way which is not particularly useful for our purposes.
A very simple method of evaluation is, however, available which is suitable for our present purpose. It is applied thus. Consider the point reached by specimen 20 in figure 4. Suppose, now, that, in-

TABLE 3

$\ln \cosh q$

$q=0.00-5.00$

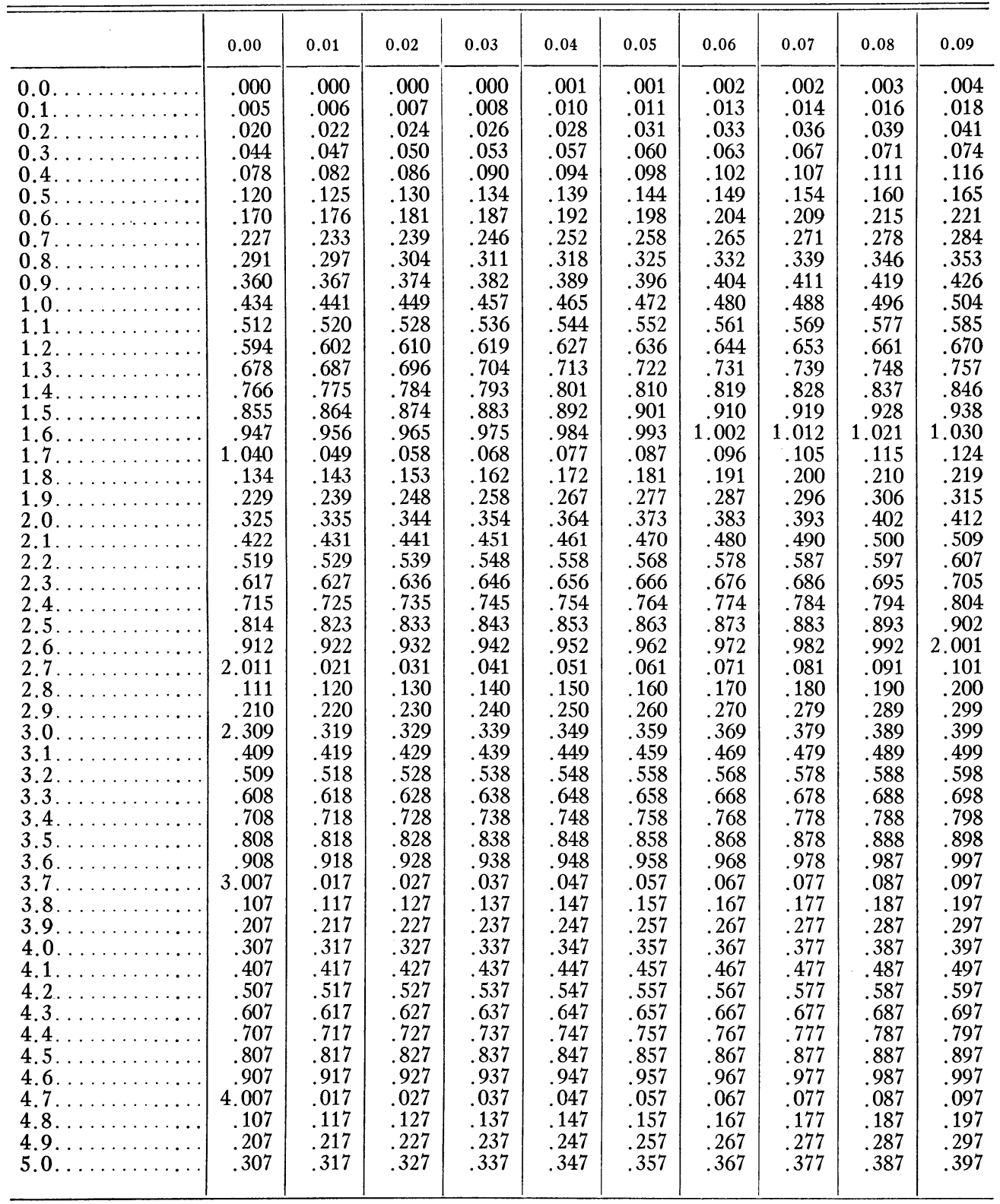


TABLE 3-Continued

$q=5.0-30.0$

\begin{tabular}{|c|c|c|c|c|c|c|c|c|c|c|}
\hline & 0.0 & 0.1 & 0.2 & 0.3 & 0.4 & 0.5 & 0.6 & 0.7 & 0.8 & 0.9 \\
\hline 5.0 . & 4.307 & .407 & .507 & .607 & .707 & .807 & .907 & $* .007$ & $* .107$ & *. 207 \\
\hline 6.0 & 5.307 & .407 & .507 & .607 & .707 & .807 & .907 & $* .007$ & $* .107$ & ${ }^{*} .207$ \\
\hline 7.0 & 6.307 & .407 & .507 & .607 & .70 & .807 & .907 & $* .007$ & $* .107$ & $* .207$ \\
\hline 8.0 & 7.307 & .407 & .507 & .607 & .707 & .807 & .907 & $* .007$ & $* .107$ & *. 207 \\
\hline 9.0 . & 8.307 & .407 & .507 & .607 & .707 & .807 & .907 & $* .007$ & $* .107$ & *. 207 \\
\hline 10.0 . & 9.307 & .407 & .507 & .607 & .707 & .807 & .907 & $* .007$ & $* .107$ & *. 207 \\
\hline 11.0 . & 10.307 & .407 & .507 & .607 & 70 & .807 & .907 & $* .007$ & $* .107$ & *. 207 \\
\hline 12.0. & 11.307 & .407 & .507 & .607 & .707 & .807 & .907 & $* .007$ & $* .107$ & $* .207$ \\
\hline 13.0 . & 12.307 & .407 & .507 & .607 & .707 & .807 & .907 & $* .007$ & $* .107$ & $* .207$ \\
\hline 14.0 . & 13.307 & .407 & .507 & .607 & .707 & .807 & .907 & $* .007$ & $* .107$ & $* .207$ \\
\hline 15.0 . & 14.307 & .407 & .507 & .607 & .707 & .807 & .907 & $* .007$ & $* .107$ & *. 207 \\
\hline 16.0 & 15.307 & .407 & .507 & .607 & .707 & .807 & .907 & $* .007$ & $* .107$ & *. 207 \\
\hline 17.0 . & 16.307 & .407 & .507 & .607 & .707 & .807 & .907 & $* .007$ & $* .107$ & *.207 \\
\hline 18.0 & 17.307 & .407 & .507 & .607 & .707 & .807 & .907 & $* .007$ & $* .107$ & $* .207$ \\
\hline 19.0 . & 18.307 & .407 & .507 & .607 & .707 & .807 & .907 & $* .007$ & $* .107$ & $* .207$ \\
\hline 20.0 & 19.307 & .407 & .507 & .607 & .707 & .807 & .907 & $* .007$ & $* .107$ & *. 207 \\
\hline 21. & 20.307 & .407 & .507 & .607 & .70 & .807 & .907 & $* .007$ & $* .107$ & *. 207 \\
\hline 22.0 & 21.307 & .407 & .507 & .607 & .707 & .807 & .907 & $* .007$ & $* .107$ & $* .207$ \\
\hline 23.0 & 22.307 & .407 & .507 & .607 & .707 & .807 & .907 & $* .007$ & $* .107$ & $* .207$ \\
\hline 24.0 . & 23.307 & .407 & .507 & .607 & .707 & .807 & .907 & $* .007$ & $* .107$ & $* .207$ \\
\hline 25.0 & 24.307 & .407 & .50 & .607 & .707 & .807 & .907 & $* .007$ & $* .107$ & *. 207 \\
\hline 26. & 25.307 & .407 & .507 & .607 & .70 & .807 & .907 & $* .007$ & $* .107$ & $* .207$ \\
\hline 27 . & 26.307 & .407 & .507 & .607 & .70 & .807 & .907 & $* .007$ & $* .107$ & $* 207$ \\
\hline 28.0 & 27.307 & .407 & .507 & .607 & .707 & .807 & .907 & $* .007$ & $* .107$ & $* .207$ \\
\hline 29.0 & 28.307 & .407 & .507 & .607 & .707 & .807 & .907 & $* .007$ & $* .107$ & *. 207 \\
\hline 30.0 & 29.307 & .407 & .507 & .607 & .707 & .807 & .907 & ${ }^{*} .007$ & $* .107$ & *. 207 \\
\hline
\end{tabular}

For values greater than those given in table $3, \ln \cosh q=q-0.693$.

stead of taking $\gamma$ to equal a probability value of 0.003 , we had chosen a value of $\gamma$ such that the line $y=s x+c$ had passed exactly through the point plotted for specimen 20 . In that case, specimen 20 would have been sufficient to render a decision that the two samples were unlike for the character in question, and the probability that this was a correct decision would be the value of $\gamma$ corresponding to that line.

Determining the value of $\gamma$ is rather simple. First evaluate the equation $y=$ $s x$, where $s$ for this setup is 0.4238 and $x$ is 20. This equation is the equation of the line of 50-50 probability. In this instance, $y=8.476$. We subtract this from 13.512 , the value of $\ln \cosh \left(d / \sigma^{2}\right)\left|\Sigma\left(x-m_{1}\right)\right|$ for specimen 20. The answer, 5.036, is the value of $c$ for the line $y=s x+c$, which would pass through this point. Referring, now, to table 3 , we find that $c=5.306$ corresponds to a value of $\gamma$ lying between 0.006 and 0.007 . By interpolation it is found that $\gamma=1-0.0068=0.9932=$ $P$ (the probability that the two samples are different in so far as this character is concerned.

\section{REFERENCES CITED}

Anderson, R. L., and Housman, E. E., 1942, Tables of orthogonal polynomials extended to $N-104$ : Iowa State College, Agr. Expt. Sta. Bull. 297.

Burma, B. H., 1948, Studies in quantitative paleontology: I. Some aspects of the theory and practice of quantitative paleontology: Jour. Paleon- tology, v. 22, p. 725-761.

Statistical Research Group, 1945, Sequential analysis of statistical data: Applications: New York, Columbia University Press.

Wald, Abraham, 1947, Sequential analysis: New York, John Wiley and Sons. 